\title{
An Advanced Particle Swarm Optimization Algorithm for MPPTs in PV Systems
}

\author{
Z. ERDEM* \\ Sakarya University, Department of Electrical and Electronics Engineering, Sakarya, Turkey
}

\begin{abstract}
Maximum power point trackers are in charge of absorbing the maximum potential power from the photovoltaic panels. Thus, this makes the maximum power point trackers the fundamental parts of the photovoltaic panel systems. The conventional maximum power point tracker algorithms are working well under balanced insolation conditions, however when the partial shade condition occurs, those algorithms are trapped at the local maxima. Hence, under partial shade conditions, the need for a global maximum power point tracking algorithm arises. Particle swarm optimization is a preferential algorithm of maximum power point trackers in literature, especially in partial shade conditions. This paper is focused on improving the existing particle swarm optimization algorithm for maximum power point trackers. The proposed advanced particle swarm optimization algorithm aims to catch the global maximum power point much faster, accurately and to reduce the chatter in the power curve. The proposed method accelerates the global maximum tracking time with gridding the initial search area. The effectiveness of the proposed method is demonstrated with simulation results and these results are compared with a conventional particle swarm optimization method under step changes in irradiance and partial shade conditions of an array of photovoltaic panels.
\end{abstract}

DOI: 10.12693/APhysPolA.132.1134

PACS/topics: 87.55.de, 88.40.mp, 87.55.kd

\section{Introduction}

In the last decade, the dramatic decrease in the amount of fossil fuels and the great demand of energy, have uncovered the significance of the renewable energy utilization on Earth. Researchers have started to investigate how to set-up high efficiency renewable energy systems with low cost $[1,2]$. At today's level of technology, photovoltaic (PV) systems still have low energy conversion efficiency rates and high costs, even though they have unlimited solar power source $[3,4]$.

Maximum power point trackers (MPPT) are some of the key parts of the PV systems, aimed to increase the efficiency of the systems [5]. Besides extracting the maximum power, generated by the PV modules, the crucial task of the MPPTs is to detect the maximum power point accurately, while the atmospheric conditions are changing [6].

Conventional single-stage tracking algorithms, that are generally based on "hill climbing", technique, like perturb and observe, incremental conductance, short circuit current etc., are implemented in several studies [7-9], however these algorithms are ineffective under partial shade conditions (PSC). The reason behind this is that when the PSC occurs, PV modules receive varying insolation, due to a chimney on the roof, or clouds, passing in front of the Sun, more than one maximum power points are formed in the output power curve.

Conventional algorithms are trapped at the local maxima, when a PSC occurs. To overcome this problem,

\footnotetext{
*e-mail: zekiye@sakarya.edu.tr
}

different global search algorithms for MPPTs are presented in the literature $[1,5,6,10-14]$. With the easy implementation and effective global search behaviour, particle swarm optimization (PSO) method is one of the preferred algorithms in MPPT systems in literature $[5,10,15,16]$. Various studies in literature are devoted to the search of the global maximum at PSC. These studies could be divided into two general groups; (i) modified conventional algorithms $[1,14,17]$, and (ii) optimization methods $[5,10-13,16,18,19]$.

PSO is a meta-heuristic algorithm and could be assigned to the group of optimization methods. The PSO algorithm was first implemented in a MPPT system by Miyateke et al. [20]. Authors have investigated the performance of the PSO algorithm under PSC and the results show that PSO is capable to carry out the global MPP search in the PV systems power output, under PSC.

After this study, the PSO-based MPPT applications have spread rapidly in literature. In study [19], a specific equation is determined to change the magnitude of a voltage shift for each agent to obtain a higher performance. Study [15] improves the PSO algorithm by using the inverse tangent function to keep the particle speed in a safe margin. Paper [11] adopts an adaptive inertial weight particle swarm optimization algorithm to improve the MPPT capability for PV systems.

In this study, original PSO algorithm is improved by selecting the initial particle positions by gridding the PV current range. This improvement increases the PSO search time, reduces the chatter range and accurately catches the global power maximum. These improvements are demonstrated using the results, obtained in MAT$\mathrm{LAB} /$ Simulink, and using comparison tables, which are given in the related sections. 


\section{PV module characteristics under PSC}

In a PV system, PV modules are built by series and parallel connection of the PV cells, and similarly, a PV array consists of PV modules, connected in series or in parallel. In case of uniform insolation, the total maximum output power of a PV array is equal to the sum of the maximum power values of all individual modules. Under PSC, the power of shaded modules decreases and the output power curve of the PV array is starting to form different levels of maximum power points. In this section, in order to explain the partial shade effect, first the characteristic of the PV array model is given, and secondly the effect of PSC is shown by simulations.

\subsection{PV array model}

An equivalent single-diode model of a PV cell is given in Fig. 1. The symbols in the circuit are defined as; $I_{\mathrm{ph}}$ is PV current source; $I_{\mathrm{D}}$ is diode current; $I_{\mathrm{SH}}$ is parallel resistance current, $R_{\mathrm{SH}}$ represents the leakage across the p-n junction, which is required to be as high as possible; $R_{\mathrm{S}}$ is the sum of resistances due to all components that come in the path of current, and which is required to be as low as possible; $I_{\text {cell }}$ is output current of PV cell; $V_{\text {cell }}$ is output voltage of the PV cell; $G$ is insolation level; $T$ is cell temperature [21].

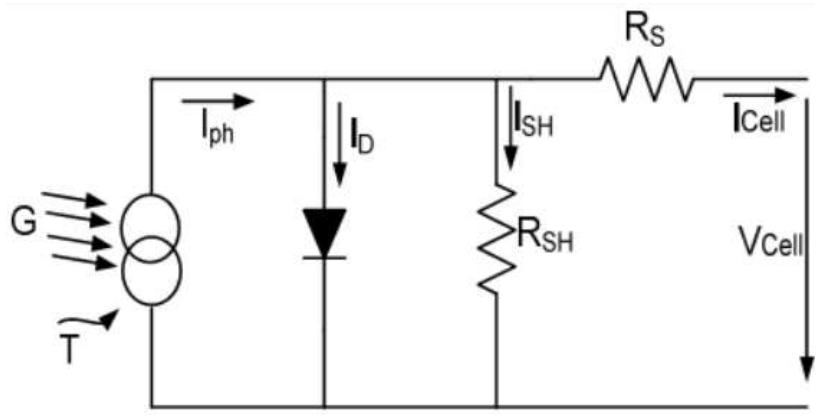

Fig. 1. Equivalent circuit of a PV cell.

$$
\begin{aligned}
& I_{\text {cell }}=I_{\mathrm{ph}}-I_{0}\left[\exp \left(\frac{q V_{\text {cell }}+q R_{\mathrm{S}} I_{\text {cell }}}{N_{\mathrm{s}} k_{\mathrm{S}} T a}-1\right)\right. \\
& \left.-\frac{V_{\text {cell }}+R_{\mathrm{S}} I_{\text {cell }}}{R_{\mathrm{SH}}}\right],
\end{aligned}
$$

where $I_{0}$ is the saturation current, $a$ is the diode factor, $k_{\mathrm{s}}$ is Boltzmann's constant, $q$ is electron charge and $N_{\mathrm{s}}$ is number of cells in series. The output current of PV module is denoted as $I_{\mathrm{pv}}$ and it depends on PV cells connected in parallel. Similarly, the output voltage of $\mathrm{PV}$ module is denoted as $V_{\mathrm{pv}}$ and it depends on PV cells connected in series.

\subsection{Effect of PSC on PV array}

A scheme of PV array and a photograph of an array under PSC are given in Fig. 2, where $m$ is the number of modules in each branch and $n$ is the number of parallel branches in the array. As shown in Fig. 2a, the modules are under different level of insolation due to shading effect.
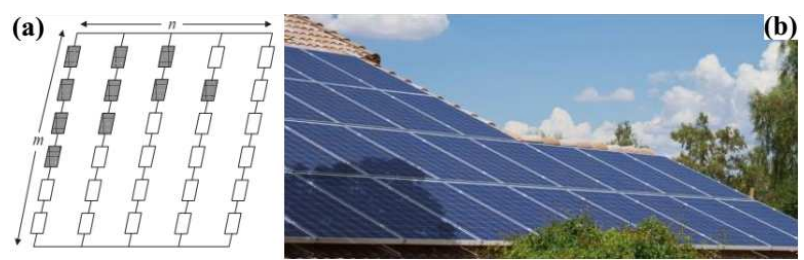

Fig. 2. (a) The schematic model of a PV array under PSC and (b) real PSC in a PV array.

In this paper, the PV array, simulated under PSC, is arranged with four PV modules as follows: $P_{\max }=85 \mathrm{~W}$; $V_{\mathrm{oc}}=22.2 \mathrm{~V} ; I_{\mathrm{sc}}=5.45 \mathrm{~A} ; V_{\max }=17.2 \mathrm{~V} ; I_{\max }=$ $4.95 \mathrm{~A}$.
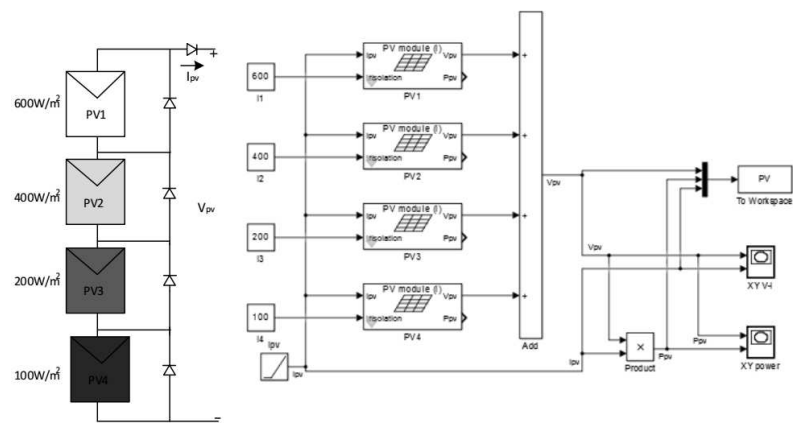

Fig. 3. MATLAB simulation of a PV array under PSC.

The simulated output characteristics of the PV array from Fig. 3, investigated under PSC, are shown in Fig. 4. Multiple peaks, i.e, local and global maxima points, are observed in the $P-V$ curve due to existence of bypass diodes.

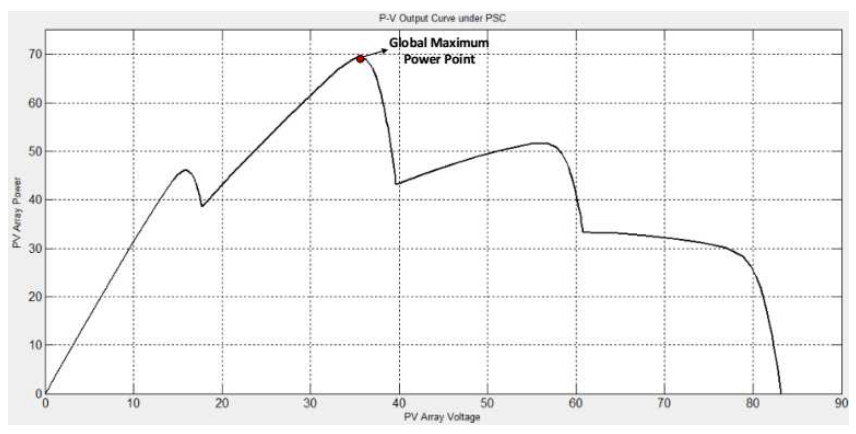

Fig. 4. $\quad P-V$ curve under PSC.

\section{Proposed advanced PSO method}

Inspired by the flocking and schooling patterns of birds and fish, PSO was invented by Russell C. Eberhart and James Kennedy in 1995 [22]. Originally, these authors 
started out by developing computer software simulations of birds flocking around food sources. They have later realized that their model was capable of solving optimization problems.

In the last decade, PSO became one of the most useful and popular algorithms in the literature to solve various optimization problems. PSO is a global searching algorithm. The optimized searching can be guided intelligently through cooperation and competition among the groups of particles. Researchers have started to use the PSO algorithm to solve the global maximum power problem, under partial shade conditions. In this section, the conventional PSO algorithm in MPPT applications is introduced. After that the improvements in the advanced PSO algorithm are described.

\subsection{Conventional PSO algorithm in MPPT systems}

PSO is an intelligent optimization algorithm. It belongs to a class of optimization group, known as metaheuristics. PSO is based on paradigm of swarm intelligence and it is inspired by the social behaviour of animals, like fish and birds. In the structure of PSO, two basic principles are used: communication and learning. In the swarm intelligence, particles cooperate to find the global optimum of the problem. PSO contains a population of candidate solutions called a swarm. Every particle of the swarm is a candidate solution to the optimization problem. Any particle has a position in the search space and this space is the set of all possible solutions to the optimization problem.

Particle $i$ has a position vector denoted by $\overrightarrow{x_{i}}(t)$. In addition to position, every particle has a velocity vector, which is denoted by $\overrightarrow{v_{i}}(t)$. The velocity describes the movement of a particle $i$ in the sense of direction and distance of the step size. In addition to position and velocity, every particle has a memory of its own best solution, as denoted by personal best " $P_{\text {best } i}(t)$ " and a global best experience among the members of swarm is denoted by $G(t)$. By defining these concepts, on every iteration of PSO, velocity and position are updated according to Eq. (2) and Eq. (3) respectively.

$$
\begin{gathered}
\underbrace{v_{i}(t+1)}_{\text {velocity of particle } i \text { at time } k+1}=\underbrace{w v_{i}(t)}_{\text {inertia }} \\
+\underbrace{r_{1} c_{1}\left(P_{\text {best } i}(t)-x_{i}(t)\right)}_{\text {cognitive component }}+\underbrace{r_{2} c_{2}\left(G(t)-x_{i}(t)\right)}_{\text {social component }},
\end{gathered}
$$

where $w$ is inertia coefficient, $r_{1}, r_{2}$ coefficients are uniformly distributed in the range of $[0,1]$ and $c_{1}, c_{2}$ are simply the acceleration coefficients.

$$
x_{i}(t+1)=x_{i}(t)+v_{i}(t+1) .
$$

Two factors are important in the design of PSO-based MPPT algorithm; (i) number of particles and (ii) convergence.

Number of particles is important for the functionality of the algorithm; a large number of particles leads to sluggish performance. On the other hand, it should be large enough to allow for implementation of PSO principles.
To find a trade-off, four particles are considered in this research.

In this research, the position of the particle is the reference current for MPPT. An initial value of current is chosen, and the corresponding PV output power is observed. Since the reference current value cannot accept any value outside the interval of $[0.2,4.95]$, the particles have to converge within these limits. If

$$
P\left(I_{\text {ref } i}(t+1)\right)>P\left(I_{\operatorname{ref} i}(t)\right),
$$

where $P\left(I_{\text {ref } i}(t)\right)$ is previous power and $P\left(I_{\text {ref } i}(t+1)\right)$ is new power, then

$$
\begin{aligned}
& P_{\text {best } i}(t)=I_{\text {ref } i}(t+1), \\
& G(t)=\max \left(I_{\operatorname{ref} i}\right), i=1,2, \ldots, N_{\mathrm{p}}, \\
& I_{\operatorname{ref} i}(t+1)=w v_{i}(t)+r_{1} c_{1}\left(P_{\text {best } i}(t)-I_{\operatorname{ref} i}(t)\right) \\
& \quad+r_{2} c_{2}\left(G(t)-I_{\operatorname{ref} i}(t)\right) .
\end{aligned}
$$

In this form algorithm can be easily implemented in a MPPT system.

\subsection{The proposed advanced PSO algorithm}

PSO is a simple yet powerful algorithm for finding the global maximum power points in MPPTs. However, in some cases, such as rapid change in insolation or partial shade conditions, conventional PSO algorithm could cause large range chattering in power and be slow in finding the global MPP. The initialization part of particle positions has a critical role in fixing these kinds of problems.

Author suggested an initialization method for hastening the algorithm and for reduction of the chattering range. In conventional PSO algorithm, applied in MPPT systems, particle positions are selected randomly, however in PV systems, the limit values of the particles are known. Therefore, the initial positions of the particles could be determined accurately, rather than randomly. In this proposed method, the limit values of the particles are linearly gridded at equal intervals and particles are placed in these determined gridded initial positions.

First the initial particle vector of reference currents is determined,

$$
\text { Initial } \vec{x}_{i}(t)=I_{\text {ref } i}(t)=\left[I_{\text {ref } 1}, I_{\text {ref } 2}, \ldots, I_{\text {ref } j}\right],
$$

$$
j=1,2, . ., N_{\mathrm{p}},
$$

where $N_{\mathrm{p}}$ indicates the number of particles.

Second the limits for each particle are determined. $\left[x_{i \text { min }}, x_{i \text { max }}\right]$ were denoted for the limits of the values of particles, which depend on the capacity of PV module. In this case, two similar PV modules (Shell Solar Q85) are connected in parallel. From the datasheet of the PV modules, the limit range of each particle is determined to be between $[1,5]$. Number of particles for each $\mathrm{PV}$ module is selected as $N_{\mathrm{p}}=4\left(R=N_{\mathrm{p}}\right)$. For gridding the initial particle position vector Eq. (10) was used and Fig. 5 explains how it works. 
$\Delta x_{i}=x_{i \max }-x_{i \min }$,

(9)

Initial $\vec{x}_{1}(t)=\left[x_{1 \min }, x_{1 \min }+\frac{\Delta x_{1}}{R}, x_{1 \min }+\frac{2 \Delta x_{1}}{R}\right.$,

$$
\left.\ldots, x_{1_{\min }}+\frac{n_{i} \Delta x_{1}}{R}\right], n_{i}=1,2 \ldots . N_{\mathrm{p}} .
$$

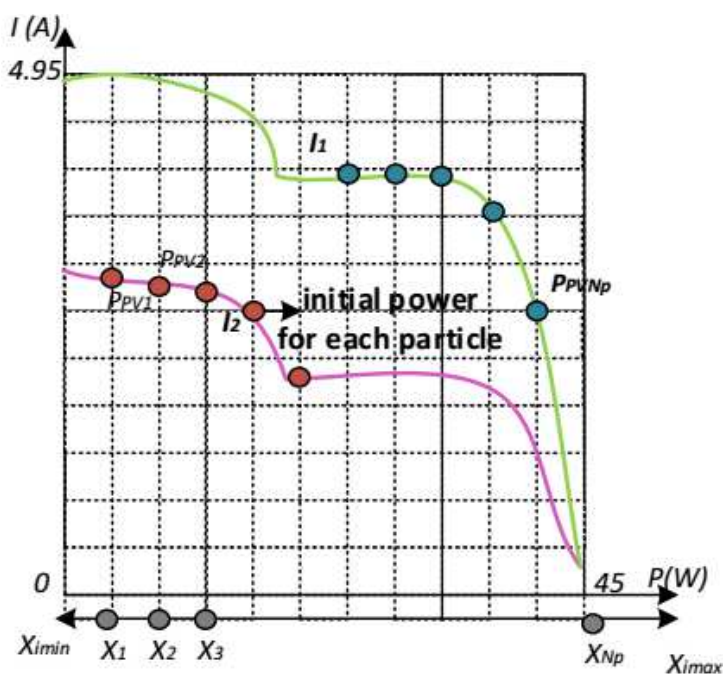

Fig. 5. Initial particle and power positions in $I-P$ curve and linear gridded search surface under different PSC.

In Fig. 5, the $I-P$ linear gridded search surface and initial positions of each particle are illustrated, with corresponding power values. The PSC conditions for $I_{1}$ and $I_{2}$ insolation levels in Fig. 5 are given in Table I.

\section{TABLE I}

PSC conditions for $I_{1}$ and $I_{2}$.

\begin{tabular}{c|c|c}
\hline \hline PV module No. & $I_{1}$ & $I_{2}$ \\
\hline PV1 & $600 \mathrm{~W} / \mathrm{m}^{2}$ & $800 \mathrm{~W} / \mathrm{m}^{2}$ \\
PV2 & $400 \mathrm{~W} / \mathrm{m}^{2}$ & $1000 \mathrm{~W} / \mathrm{m}^{2}$
\end{tabular}

The flowchart of the proposed advanced PSO algorithm is given below and the contribution to the conventional PSO algorithm is shown in the highlighted box. In this proposed flowchart, the algorithm starts with determining the initial particle population size, which is denoted as $N_{\mathrm{p}}$. After that, the initial particle locations are determined based on Eq. (6), which grids the search space. The other procedures are completely the same as in the conventional PSO algorithm.

\section{Simulation results}

The proposed advanced PSO algorithm allows to increase the total power obtained form PV array at different insulation levels and to reduce the chance of the algorithm to be trapped at the local maxima. The results are shown in this section.

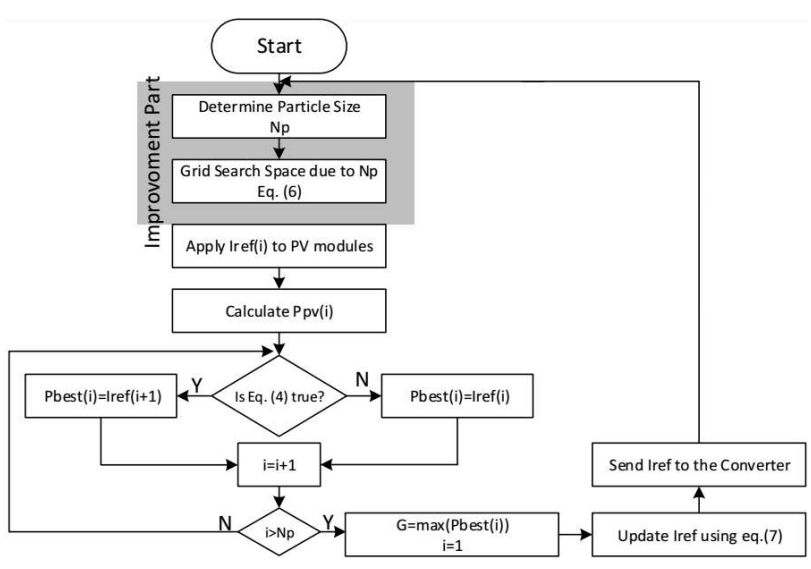

Fig. 6. Flowchart of the advanced PSO algorithm.

Figure 7 shows the MATLAB-Simulink simulation model of the PV system used in this study. To implement the PSO algorithm, the following control parameters were used in both simulation and implementation: $C_{1}=0.8, C_{2}=0.5$.

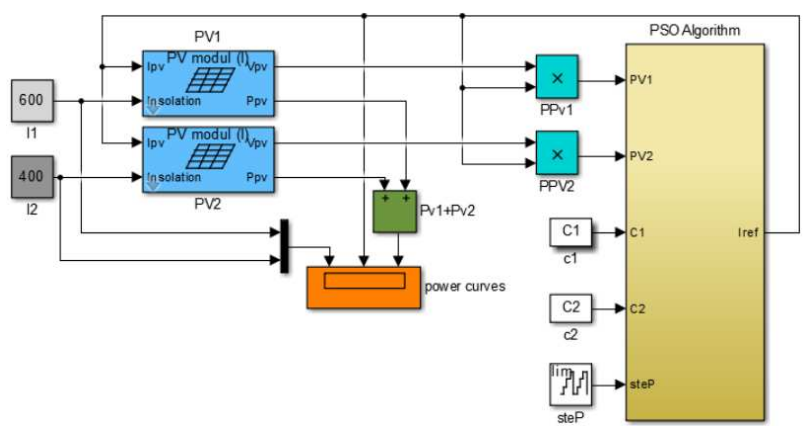

Fig. 7. MATLAB-Simulink simulation model for the PV system.

After the simulations of proposed PSO algorithm on Simulink model, shown in Fig. 7, the output power curve of the PV array is given in Fig. 8a and the particle movement under PSC is shown in Fig. 8b. Different insolation conditions are applied through the simulation. In the first scenario; $\mathrm{PV}$ module $1\left(\mathrm{PV}_{1}\right)$ is under $500 \mathrm{~W} / \mathrm{m}^{2}$ $\left(I_{1}\right)$ and $\mathrm{PV}$ module $2\left(\mathrm{PV}_{2}\right)$ is under $600 \mathrm{~W} / \mathrm{m}^{2}\left(I_{2}\right)$ insolation level. In the second scenario, $I_{1}=400 \mathrm{~W} / \mathrm{m}^{2}$, $I_{2}=900 \mathrm{~W} / \mathrm{m}^{2}$. In the last scenario, $I_{1}=865 \mathrm{~W} / \mathrm{m}^{2}$, $I_{2}=340 \mathrm{~W} / \mathrm{m}^{2}$.

In Fig. 9, the performance of the advanced PSO algorithm is compared with the conventional PSO algorithm under equal PSC. PV output power curves for both algorithms and maximum power curve, which indicates the theoretical power, are shown in Fig. 9. The PV system was exposed to the uniform insolation, with insolation rates of $\mathrm{PV}_{1}=490 \mathrm{~W} / \mathrm{m}^{2}$ and $\mathrm{PV}_{2}=860 \mathrm{~W} / \mathrm{m}^{2}$.

A comparison table is given in Table II. The performance of initially random particles and proposed gridbased particles is compared using initial positions, settling time, maximum power point and chattering range. 

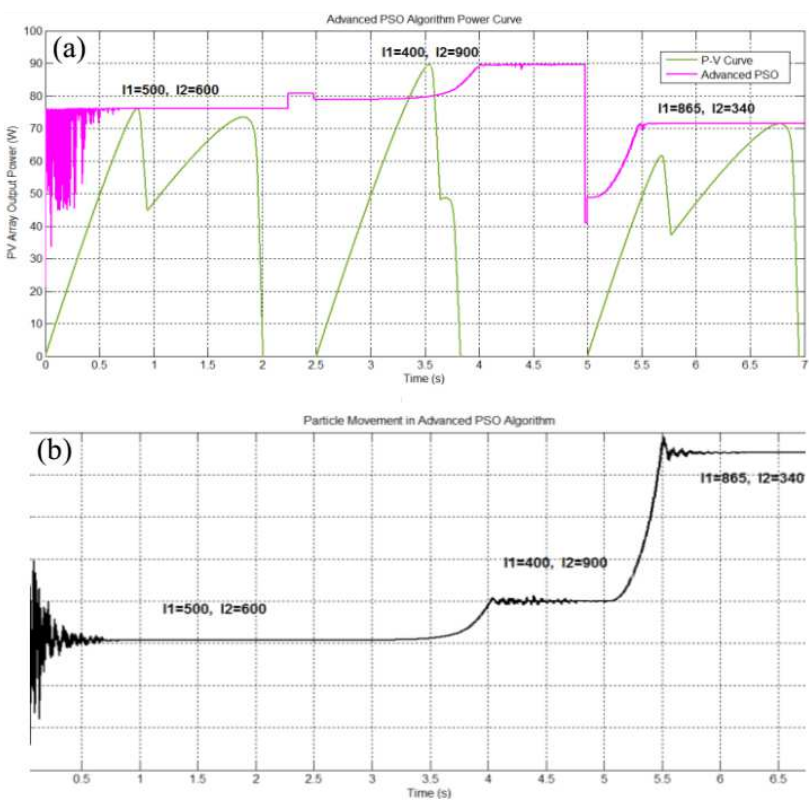

Fig. 8. (a) PV output power for advanced PSO algorithm. (b) Particle $I_{\text {ref }}$ movement at different insolation levels.

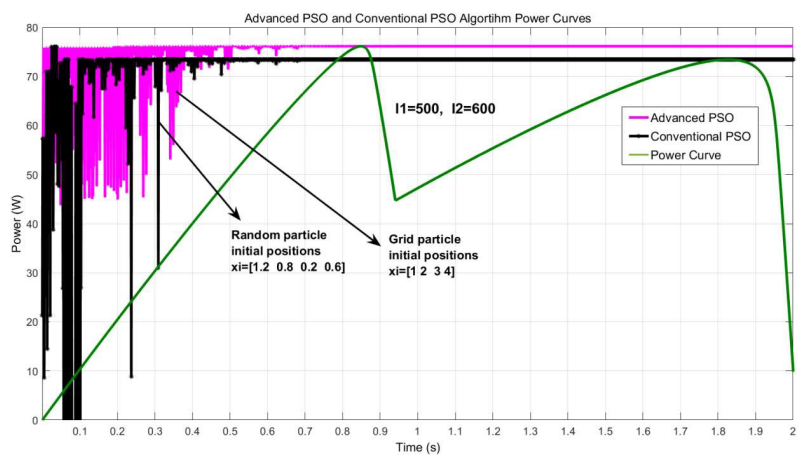

Fig. 9. PV output power curves of advanced and conventional PSO algorithm.

The selection of random positions for initial particles causes a large number of cases. Due to this, one of the worst case scenarios is selected for comparison and is shown in Fig. 9. As clearly shown in Fig. 9; the conventional PSO algorithm is trapped at local maxima and causes large chattering in the power.

TABLE II

Comparison table of results of PSO and advanced PSO algorithm.

\begin{tabular}{c|c|c}
\hline \hline Compared value & Random particles & Grid particles \\
\hline Initial positions & {$\left[\begin{array}{llll}1.2 & 0.8 & 0.2 & 0.6\end{array}\right]$} & {$\left[\begin{array}{lll}1 & 2 & 3\end{array}\right]$} \\
Settling time [s] & 0.512 & 0.485 \\
Maximum power [W] & 73.42 & 76 \\
Chattering range [W] & 76 & 30
\end{tabular}

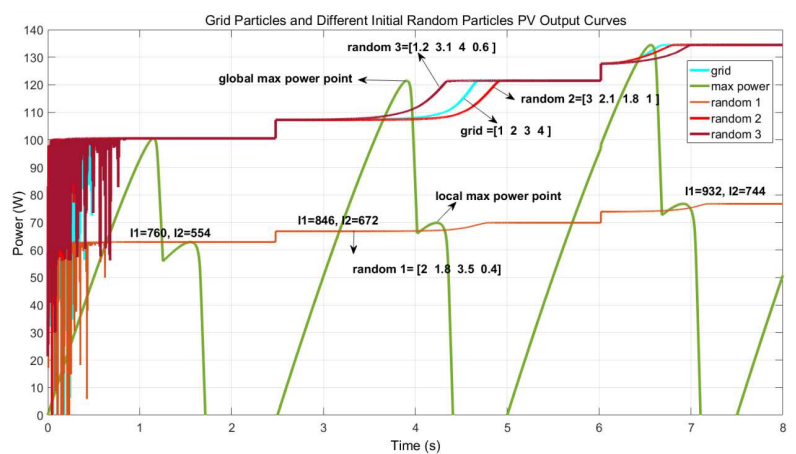

Fig. 10. PV output curves for grid particles and different random particles.

Figure 10 shows the performance of an alternative random position, compared to the proposed grid particles at different insolation step changes. Three different random particles are implemented in the PV system and the results show the importance of the initial particle positions.

As an example, if the designer unconsciously selected randomly the initial particle position 1, the algorithm will be trapped at the local maxima at every PSC. At first sight, the random case 3 shows a better performance than the grid particles. However, the chattering range in this case is larger than for grid particles. The comparison table for power curves from Fig. 10 and insolation rates are given in Tables III and IV, respectively.

TABLE III

Comparison table of random particles and grid particles.

\begin{tabular}{c|c|c|c|c}
\hline \hline $\begin{array}{c}\text { Compared } \\
\text { value }\end{array}$ & Random 1 & Random 2 & Random 3 & $\begin{array}{c}\text { Grid } \\
\text { particles }\end{array}$ \\
\hline $\begin{array}{c}\text { Initial } \\
\text { positions }\end{array}$ & {$\left[\begin{array}{llll}2 & 1.8 & 3.5 & 0.4\end{array}\right]$} & {$\left[\begin{array}{llll}3 & 2.1 & 1.8 & 1\end{array}\right]$} & {$\left[\begin{array}{llll}1.2 & 3.1 & 4 & 0.6\end{array}\right]$} & {$\left[\begin{array}{lll}1 & 2 & 3\end{array}\right]$} \\
$\begin{array}{c}\text { Maximum } \\
\text { power [W] }\end{array}$ & $63-70-76$ & $100-121-134$ & $100-121-134$ & $100-121-134$ \\
$\begin{array}{c}\text { Chattering } \\
\text { range [W] }\end{array}$ & 62.5 & 100 & 100 & 70
\end{tabular}

TABLE IV

Insolation rates in Fig. $10\left(\mathrm{~W} / \mathrm{m}^{2}\right)$.

\begin{tabular}{c|c|c|c}
\hline \hline Time range & $0-2.48 \mathrm{~s}$ & $2.48-6 \mathrm{~s}$ & $6-8 \mathrm{~s}$ \\
\hline $\mathrm{PV}_{1}$ insolation $\left(I_{1}\right)$ & 760 & 846 & 932 \\
$\mathrm{PV}_{2}$ insolation $\left(I_{2}\right)$ & 554 & 672 & 744
\end{tabular}

\section{Conclusions}

This paper presents an advanced PSO algorithm for MPPT systems under PSC. The proposed method involves a modification of the conventional PSO algorithm to improve its performance. The conventional PSO algorithm has some drawbacks, like trapping at the local 
maxima and large chattering power range. The proposed algorithm is aimed to eliminate these drawbacks by linear gridding the initial particle positions. Proposed algorithm was simulated in the MATLAB environment with various PSCs and comparison of simulation results shows that the proposed algorithm tracks the true global maximum point more accurately, faster and significantly reduces the chattering range of the power.

\section{References}

[1] S. Choudhury, in: India Conference (INDICON), 2015 Annual IEEE, IEEE, 2015, p. 1.

[2] Z. Er, I.B. Turna, Acta Phys. Pol. A 129, 865 (2016).

[3] M. Aghaei, A. Dolara, F. Grimaccia, S. Leva, D. Kania, J. Borkowski, in: 2016 IEEE 16th Int. Conf. Environment and Electrical Engineering (EEEIC), IEEE. 2016, p. 1.

[4] B. Kiriş, O. Bingöl, R. Senol, A. Altintaş, Acta Phys. Pol. A 130, 55 (2016).

[5] Z. Yibin, W. Weiying, C. Weirong, L.I. Qi, in: 2016 35th Chinese Control Conf. (CCC), IEEE, Chengdu 2016, p. 8654.

[6] T.T. Yetayew, in: 2016 IEEE 6th Int. Conf. Power Systems (ICPS), IEEE, New Delhi 2016, p. 1.

[7] M.I. Bahari, P. Tarassodi, Y.M. Naeini, A.K. Khalilabad, P. Shirazi, in: 2016 Int. Symp. Power Electronics, Electrical Drives, Automation and Motion (SPEEDAM), IEEE, Anacapri 2016, p. 1041.

[8] W. Rahman, C. Bathina, V. Karthikeyan, R. Prasanth, in: 2016 10th Int. Conf. Intelligent Systems and Control (ISCO), IEEE, Coimbatore 2016, p. 1.

[9] Z.M. Dalala, Z.U. Zahid, in: 2015 IEEE Energy Convers. Congr. Expo. ECCE, IEEE, 2015, p. 1062.
[10] K. Ishaque, Z. Salam, M. Amjad, S. Mekhilef, IEEE Trans. Power Electron. 27, 3627 (2012).

[11] X. Yuan, D. Yang, H. Liu, in: 2015 IEEE Int. Conf. Cyber Technol. Autom. Control. Intell. Syst., IEEE, 2015, p. 729 .

[12] S. Mohanty, B. Subudhi, P.K. Ray, IEEE Trans. Sustain. Energy 7, 181 (2016).

[13] I. El Jazzar, Appl. Soft. Comput. 32, 38 (2015).

[14] R. Alik, A. Jusoh, N.A. Shukri, in: 2015 IEEE Conf. Energy Conversion, CENCON, IEEE, 2016, p. 398.

[15] V. Phimmasone, Y. Kondo, N. Shiota, M. Miyatake, in: 2013 1st Int. Futur. Energy Electron. Conf., IEEE, Taiwan 2013, p. 81.

[16] S.M. Mirhassani, M. Razzazan, A. Ramezani, in: 22nd Iran. Conf. Electr. Eng. ICEE 2014, IEEE, Tehran 2015, p. 550

[17] Q. Duan, J. Leng, P. Duan, B. Hu, M. Mao, in: 2015 Yth Int. Conf. Intelligent Human-Machine Systems and Cybernetics (IHMSC), vol. 1, IEEE, Hangzhou 2015, p. 382.

[18] E. Malarvizhi, in: 2016 10th Int. Conf. Intelligent Systems and Control (ISCO), IEEE, Coimbatore 2016, p. 1.

[19] V. Phimmasone, Y. Kondo, T. Kamejima, M. Miyatake, in: Electr. Mach. Syst. (ICEMS), 2010 Int. Conf., IEEE, Incheon 2010, p. 487.

[20] M. Miyatake, M. Veerachary, F. Toriumi, N. Fujii, H. Ko, IEEE Trans. Aerosp. Electron. Syst. 47, 367 (2011).

[21] A.A. Jadallah, D.Y. Mahmood, Z.A. Abdulqader, Acta Phys. Pol. A 128, B-461 (2015).

[22] J. Kennedy, R. Eberhart, in: Proc. Neural Networks, IEEE Int. Conf., vol. 4, IEEE, 1995 p. 1942 